\title{
VEINTICINCO AÑOS DE CONSTITUCIÓN Y FUERZAS ARMADAS
}

\author{
GUSTAVO SUÁREZ PERTIERRA \\ Catedrático de Derecho Eclesiástico del Estado \\ UNED
}




\section{SUMARIO}

UNA CONSTITUCIÓN PARA LA SOLUCIÓN DE UN PROBLEMA HISTÓRICO. - LAS BASES CONSTITUCIONALES DE LA REFORMA MILITAR. - LA INSTITUCIONALIZACIÓN DE LA DEFENSA. - LA INTEGRACIÓN DE LA DEFENSA EN LOS PODERES DEL Estado. OBJeTIVOS DE DEFENSA Y MISIONES DE LAS FUERZAS ARMADAS. - EL CAMBIO DEL ESCENARIO ESTRATÉGICO. - La CONSTITUCIÓN, MOTOR de La REFORMA. UNA CONSTITUCIÓN ABIERTA. 


\title{
VEINTICINCO AÑOS DE CONSTITUCIÓN Y FUERZAS ARMADAS
}

\author{
POR \\ GUSTAVO SUÁREZ PERTIERRA \\ Catedrático de Derecho Eclesiástico del Estado \\ UNED
}

\section{UNA CONSTITUCIÓN PARA LA SOLUCIÓN DE UN PROBLEMA HISTÓRICO}

Nadie discute que la Constitución española ha sido un hito fundamental en la historia de nuestra transición. Tampoco que dicha consideración deba extenderse al tratamiento de aquellas cuestiones que tienen que ver con uno de sus núcleos fundamentales: las Fuerzas Armadas. Precisamente uno de los méritos que se atribuyen al texto de 1978 es el de haber superado una cuestión endémica en España: el llamado problema militar. Durante buena parte de los siglos XIX y XX, con pocos paréntesis y al amparo de la consideración de institución especial que las leyes les otorgan, los ejércitos españoles han venido desarrollando un protagonismo que les sitúa como una organización privilegiada para la tutela del sistema.

La transición a la democracia se lleva a cabo desde un sistema autoritario en el que las Fuerzas Armadas, aunque no son los actores principales, constituyen la garantía de su sostenimiento. Se comportaron a lo largo de varias décadas como una organización dotada de 
amplia autonomía y con indudable influencia social propia. Esta es la clave de arco del problema militar a la llegada de la democracia, porque la reforma militar ha sido en mi opinión, antes que otra cosa, el proceso de ubicación de los ejércitos en el entramado de las instituciones del Estado de Derecho que define la propia Constitución.

La base principal para el proceso de incorporación de las fuerzas armadas al sistema político democrático es la Constitución de 1978. El proceso fue largo y se desarrolló en diversos frentes y a través de medidas complejas de orden diverso. No está de más intentar una explicación de dicho proceso a la hora de realizar un balance de los veinticinco años de vigencia del texto constitucional. Este escrito pretende ofrecer una reflexión sobre el alcance del camino recorrido, que la Constitución ampara. Seguramente requeriría profundización en algunos de los argumentos que se presentan, que en parte he llevado a cabo en otras publicaciones. Pero hoy se trata de presentar una reflexión panorámica esencial.

Muchas cosas han cambiado en este tiempo en la sociedad española, pero también en el mundo sin fronteras que estamos viviendo. Entre otras cuestiones, ha cambiado el papel de unos ejércitos que en la tradición continental fueron concebidos como un elemento primordial de vertebración de las sociedades nacionales y que ahora incorporan la completa profesionalización. También ha cambiado el propio concepto de seguridad. Irrumpen para las fuerzas armadas nuevas misiones que no estaban pensadas hace siquiera poco más de una década y quién sabe si estamos ante un cambio de naturaleza de la idea misma de soberanía, tradicionalmente asentada sobre unos pilares que han entrado en crisis.

Pero todo esto ha pasado después. La sociedad española ha podido enfrentarse a la nueva situación desde una posición suficientemente asentada en la modernidad. La Constitución supuso dentro de este ámbito, en mi criterio, la palanca para la resolución del viejo problema histórico y el cauce para la aplicación progresiva del proceso de reforma.

\section{LAS BASES CONSTITUCIONALES DE LA REFORMA MILITAR}

La propia Constitución es un hito de la transición política española. En materia militar ya se habian producido actuaciones de interés antes de 1978 que cumplieron la función histórica de ir preparando la reforma. Cuando el General GUTIÉRREZ MELLADO da prioridad 
a la elaboración de unas Reales Ordenanzas para las Fuerzas Armadas que vengan a sustituir las viejas Ordenanzas de Carlos III, lo hace señalando el camino de la reforma, pero también para poner de manifiesto que al Gobierno interesa acometer sin demora la reforma militar. La propia norma citada enfrenta aspectos sometidos a gran polémica, como el de la obediencia debida, y se emprenden actuaciones de calado, como la regulación de la participación de los militares en la política o la creación de un Ministerio de Defensa ya en 1977. Con todo, no es menos cierto que la iniciativa sobre las Ordenanzas fue adoptada desde el ámbito militar para adelantarse a la opción gubernativa, que hubiera sido menos manejable; también que la regulación de la participación de los militares en la política sigue pautas muy rígidas, como seguramente convenía al momento, pero que luego resultaron difíciles de conducir; e, igualmente, que el Ministerio de Defensa no existió prácticamente más que sobre el papel, porque las competencias de los tres ejércitos siguieron siendo casi las mismas y la Junta de Jefes de Estado Mayor estaba constituida como órgano supremo de la cadena de mando militar.

Sin embargo, el momento constitucional todavía coincide con el panorama de unos ejércitos acostumbrados a intervenir en la vida pública y caracterizados por sus exageradas dimensiones, sus cuadros de mando formados en la guerra civil, la falta de medios materiales $y$ el aislamiento internacional (salvo la conexión con los Estados Unidos de América después de los pactos de 1953). Es fácil representarse en este contexto las crisis, agravadas por el terrorismo, que la influencia de los acontecimientos políticos iba provocando, fundamentalmente las derivadas de la normalización de la vida política y de la implantación de la organización territorial del Estado.

Pues bien, para enfrentarse a la regulación del problema, la Constitución opta por un sistema complejo. Yo creo que deben destacarse dos cuestiones, la primera de carácter instrumental y la segunda de fondo o de contenido. La primera cuestión es el procedimiento de elaboración del texto. Lo que dio en llamarse consenso funcionó en este ámbito, como en otros de la Constitución y en todo el contexto de la transición política, de tal manera que las soluciones constitucionales son, por una parte, producto del instrumento del diálogo empleado para su elaboración, al tiempo que, de otra, buena parte de ellas no pueden entenderse sin el recurso a este criterio, que se convierte, así, en un principio hermenéutico del texto constitucional.

Esto tiene mucho que ver con la segunda cuestión. Y ello porque la regulación de la temática de las Fuerzas Armadas en el texto 
de 1978 resulta especialmente compleja. La base es doble: un supuesto en el Título Preliminar, el artículo $8^{\circ}$, y otro supuesto, el artículo 97, en el Título correspondiente al Gobierno y la Administración. La solución, por más que se intente el tratamiento aislado del primero de los preceptos, que concentra toda la importancia de la ubicación de las Fuerzas Armadas al lado de las instituciones básicas y de los fundamentos del Estado, no es posible si no se ponen en conjunto ambas regulaciones.

La regla del consenso permitió que el artículo $8 .^{\circ}$, tan sorprendente en nuestra historia constitucional, no fuera objeto de especial polémica. Tan sólo se destacaron, sin gran insistencia, algunas cuestiones relativas a su propia supresión, a la enumeración de las Fuerzas Armadas en él contenida, a la expresión de las misiones con referencia a la unidad de la nación y, precisamente, a su ligazón sistemática con el artículo 97. Parece que los constituyentes tuvieron claro desde el primer momento el planteamiento que finalmente sería aprobado.

Hay aquí un problema de base que ha sido destacado por la doctrina. Se trata de que, en la medida que la referencia constitucional a los ejércitos, favorecida por su ubicación, les consolide como una institución fundamental del Estado, con sus propios códigos de conducta y una organización peculiar, pudiera reclamar un cierto grado de autonomia, residuo de planteamientos históricos, en el conjunto de la Administración cuya dependencia del poder ejecutivo recoge el art.97.

De ahí que lo más relevante en el problema sea la conexión constitucional entre los dos supuestos fundamentales que hacen referencia a este campo. En mi criterio, lo que el artículo $8 .^{\circ}$ incorpora a través de su referencia a las Fuerzas Armadas es, más que la constitucionalización de las Fuerzas Armadas mismas, la definición constitucional de las funciones de garantía y defensa que deben ejercer. Estas misiones son las clásicas y están ligadas a los elementos básicos de la idea de soberanía del Estado, pero en ningún lugar estaban explicitadas y a menudo se habían venido confundiendo con la función de mantenimiento de la seguridad interna o el orden público administrativo y penal. Así pues, el supuesto ubicado en el Título Preliminar pretende sin más la vinculación de los ejércitos con el desarrollo de sus misiones fundamentales, que sólo a través de los propios principios constitucionales pueden ejercerse. Como quiera que la sujeción a las directrices del Gobierno está asegurada a través del artículo 97, lo que se lleva al Título Preliminar es el componente de misión o función para mejor imbricarla con los principios constitucionales. 
Por lo demás, la Constitución hace referencia en lugares paralelos a otros supuestos relacionados con los aspectos militares. Algunos de estos supuestos provienen del modelo de organización del Estado, como la declaración de la Defensa y Fuerzas Armadas como objeto de su competencia exclusiva (art. 149, 4. ${ }^{\text {a) }}$, la consideración del Jefe del Estado como mando supremo de las Fuerzas Armadas (aunque sus actos siempre habrán de ser refrendados por el Presidente del Gobierno o Ministro competente: arts. 62 . h y 64.1 ), la vinculación del servicio militar con la defensa nacional (art. 30) o la reserva a las Cortes Generales de la autorización previa de tratados y convenios de carácter militar (art. 94, 1. b). Otros supuestos hacen referencia a la limitación de los derechos fundamentales de los militares, como la sindicación o el derecho de petición individual (artcs. 28 y 29). Todos son aspectos de señalada importancia en la definición del sistema constitucional que afecta a las Fuerzas Armadas. Entre ellos, funciona directamente como elemento de contención de la autonomía militar la conexión entre principio de unidad jurisdiccional y jurisdicción militar prevista en el art. 117, 5. En sentido contrario, algunas disposiciones constitucionales parecen trabajar como elementos de compensación, que tienden a salvar dudosas especificidades militares (la excepción para los tribunales de honor: art. 26) o que guardan prudencia frente a determinadas cuestiones supuestamente más sensibles (la posibilidad del mantenimiento de la pena de muerte en las leyes militares para tiempo de guerra: art. 15). Ambas previsiones están hoy sin efecto a causa de reformas legales producidas con posterioridad.

El propio artículo $8 .^{\circ}$ hace referencia a una cuestión de entidad en su párrafo segundo. Por cuanto la Constitución sólo recoge los principios y técnicas fundamentales del sistema, la regulación de los diferentes institutos precisa ulterior desarrollo. A ello se refiere el mencionado supuesto cuando establece que Una ley orgánica regulará las bases de la organización militar.

El mandato recibe pronto la atención del legislador: en 1980 se promulga la Ley orgánica de criterios básicos de la defensa nacional y de la organización militar. Es el primer desarrollo del planteamiento constitucional, que se revisa en enero de 1984. Puede decirse que es precisamente en esta fecha y como producto de la mencionada reforma cuando se inician de manera sistemática los procesos que, tomados en conjunto, han recibido comúnmente el nombre de reforma militar. Desde entonces, la norma fundamental en la materia por debajo de la Constitución no ha sufrido alteraciones directas. La actual Revisión Estratégica, del año 2003, prevé la elaboración de un nuevo texto de Ley de criterios básicos de la Defensa Nacional que integre las 
novedades estratégicas y los cambios requeridos en la organización de la defensa y de las Fuerzas Armadas.

\section{LA INSTITUCIONALIZACIÓN DE LA DEFENSA}

Antes se ha dicho que el proceso de transición militar se concentra en el ámbito de lo que en otro lugar he llamado llamado constitucionalización de los ejércitos, que es la base y el contenido fundamental de la reforma militar. Es decir, se trata de buscar soluciones adecuadas al papel que las Fuerzas Armadas deben desempeñar en el entramado de las instituciones estatales o, si se quiere, ordenar su inserción en el marco del Estado donde los poderes clásicos deben desarrollar sus competencias en relación con la preparación y el uso de la fuerza material que el propio Estado detenta.

Este es el proceso fundamental que se ha venido sustanciando durante una buena parte de la vigencia constitucional. Conviene dejar sentadas dos precisiones como marco general de acercamiento al mencionado proceso. En primer lugar, los términos de fondo del problema, que no son otros que la determinación de los sujetos que ejercen la autoridad sobre la defensa y los procedimientos que deben emplearse para ello. Una vez definido el fundamento, es preciso decidir las funciones que los ejércitos deben cumplir en la sociedad democrática, que no son fáciles de concretar, que trabajan en zonas a veces poco delimitadas y que deben constar prefijadas en normas jurídicas. El conjunto de estos planteamientos apunta a un fenómeno que puede denominarse como institucionalización de la defensa.

Aún debe indicarse una segunda precisión. Estos procesos a menudo se ejercen sobre sociedades fuertemente desestructuradas y sometidas a tensiones de cambio, donde las instituciones públicas suelen ser todavía débiles y en las que las pocas organizaciones trabadas internamente por criterios de disciplina mantienen una disposición natural de tutela hacia la evolución del propio proceso. Todo ello hace que los procesos sean extensos en el tiempo y plantean dificultades para el mantenimiento de una tensión que, sin embargo, resulta completamente necesaria para la obtención de los resultados requeridos. $Y$ esto hace, también, que los largos procesos de reforma dependan de programas esenciales y generalmente poco estructurados, que se van concretando con el transcurso del tiempo y que permiten adaptar los ritmos a la conveniencia de su desarrollo. No suele tratarse, por tanto, de procesos sujetos a un diseño cerrado, aunque es muy importante 
que los horizontes finales se encuentren bien definidos, pues una organización de la naturaleza de la militar siempre necesita saber hacia dónde dirige su camino.

Pues bien, los desarrollos constitucionales que se emprenden fundamentalmente a partir de 1984 se perfilan con estos criterios y persiguen prioritariamente la finalidad de establecer un modelo adecuado para la integración institucional de los ejércitos y la fijación de sus misiones. Es esta la primera necesidad y se corresponde con las preocupaciones de la sociedad española. Es un proceso complejo, que se mantiene tensionado durante toda una década, aunque adapta sus ritmos a las necesidades de cada momento y que va incorporando dinámicamente las medidas que la adecuada llevanza del problema requiere.

\section{LA INTEGRACIÓN DE LA DEFENSA EN LOS PODERES DEL ESTADO}

El primer elemento, por consiguiente, es la configuración de los poderes clásicos en relación con la defensa, que tiene como punto de partida la atribución al Estado de la competencia exclusiva en la materia por prescripción del propio texto constitucional. Así pues, la capacidad de crear fuerza armada, la capacidad de organización de los ejércitos y la competencia de dirección de la función defensa queda concentrada en el Estado. Conviene advertir que todo ello se establece sin perjuicio de la idea integradora de la defensa nacional como disposición, integración y acción coordinada de todas las energías y fuerzas morales y materiales de la Nación que ya utiliza la Ley de Criterios Básicos de la Defensa Nacional en su redacción de 1980 y que obliga a la participación en la defensa de las instituciones públicas y privadas y de los ciudadanos todos.

El poder legislativo, por su parte, tiene atribuidas desde la Constitución, en este campo, las funciones generales de potestad legislativa y control del gobierno y las restantes que la propia Constitución y las leyes le atribuyen. La propia Ley de Criterios básicos, al incorporar estos parámetros, le atribuye, entre otras cuestiones, el debate de las líneas generales de la política de defensa (art. $6^{\circ}{ }^{\circ}$ ).

Desde la perspectiva de reflexión general en que estas páginas se sitúan, considero conveniente realizar algunas acotaciones. En primer lugar, aunque es verdad que nunca se han puesto en práctica algunas de las previsiones de la Ley, como la relativa al debate general 
sobre los programas de armamento y obviando en este momento los debates en materia de definición de política exterior que cada vez más tienen relación con la política de defensa, entiendo que debe hacerse referencia a la intervención parlamentaria en la definición de los grandes procesos de cambio que afectan a las fuerzas armadas.

El primer hito es de 1991, fecha en que una Comisión parlamentaria realiza un proceso de reflexión sobre las Fuerzas Armadas del fin de siglo que elabora un documento aprobado con amplio consenso. Era el primer planteamiento de fondo que se hacía en España sobre el particular en sede parlamentaria. Este informe va a dar lugar, por una parte, a la definición de un modelo de ejércitos mixto, más reducido y profesionalizado por encima del $50 \%$; de otra, una vez producida ya en 1989 la regulación de los cuadros de mando, que por primera vez en la historia abarca toda la carrera militar, la propia reflexión dará lugar a la ordenación de la figura del soldado profesional y a la aprobación de una nueva Ley de Servicio militar.

Siguiendo este modelo, pero con la sustancial diferencia de que la decisión se había adoptado previamente por un Gobierno apremiado al pacto político con la minoría, en 1996 se constituye una nueva Comisión mixta Congreso-Senado para estudiar las exigencias de la profesionalización. Su informe final, también consensuado por la mayor parte de las fuerzas políticas, dio lugar a la completa profesionalización de las fuerzas armadas españolas.

Finalmente, en otro orden de cosas, una cuestión merece la pena destacar aunque pudiera resultar para algunos obvia. Es la siguiente: el control parlamentario se ejerce fundamentalmente (y casi exclusivamente) sobre los miembros del Gobierno (en pleno y en comisiones) y sobre los altos cargos civiles del Gobierno (en comisiones). En contra de lo que sucede en otros sistemas, se ha procurado evitar el control sobre los responsables militares en el Congreso, que sólo comparecen en determinadas ocasiones a efectos de informar sobre aspectos concretos (proyecto de presupuesto). Esta circunstancia suele evitar el acceso de los grupos parlamentarios a la información concreta sobre el desarrollo de los programas de reforma y acusa la consideración de los más altos responsables militares como órganos de ejecución de carácter técnico en los términos que luego se dirá.

Durante este tiempo la reforma militar va pivotando entre la actividad del poder legislativo y la del poder ejecutivo. En mi criterio, a pesar de que la acción parlamentaria viene incidiendo en la materia con sus funciones habituales, hay un amplio margen de trabajo para el Gobierno, que lleva la iniciativa de la reforma. Lo facilita la existencia 
de una mayoría política holgada, pero también la permanencia en esta materia del consenso constitucional, la falta de cuadros políticos preparados en las cuestiones militares y el entendimiento de que el proceso de reforma debe ser acometido con criterios de prudencia. Luego se dirá que el instrumento esencial para la dirección de la reforma es el Ministerio de Defensa.

El proceso de articulación de las competencias sobre defensa en el ámbito del ejecutivo ha sido largo y cuidadoso. Se desarrolla a partir de 1984 generalmente a través de disposiciones jurídicas de carácter reglamentario que toman causa en la Ley de Criterios básicos. Como tal proceso tiene su fundamento, en mi opinión, en dos principios informadores que podrían denominarse así: distinción de competencias y personalización de las decisiones.

El primero podría configurarse en base a la distinción entre acción política y acción ejecutiva. Por su virtud, la función de dirección politica, que el art. 97 de la Constitución atribuye al Gobierno, se distribuye entre el propio Gobierno, que determina la politica de defensa y dirige la administración militar, su Presidente, que dirige la política de defensa y la actuación de las fuerzas armadas y define los grandes objetivos estratégicos, y el Ministro de Defensa, que ejerce competencias de politica militar con potestad propia y ordena, coordina y dirige la actuación de las fuerzas armadas por delegación del Presidente del Gobierno.

Las capacidades de dirección política quedan, pues, concentradas en los órganos del ejecutivo. La gran novedad que se produce en 1984 con la reforma de la Ley de Criterios básicos es justamente la integración en la jerarquía del mando militar de la figura del Presidente del Gobierno, que se superpone a una Junta de Jefes de Estado Mayor constituida hasta entonces como órgano superior de la cadena de mando militar.

Es preciso hacer referencia en este instante al que se ha definido como instrumento especialmente relevante para la reforma: el Ministerio de Defensa. Creado en 1977, tuvo una existencia poco más que formal en términos de dirección política unificada, pues los ejèrcitos mantenían buena parte de las competencias de los antiguos ministerios militares. Es también en febrero de 1984 cuando comienza a ser potenciado para hacer frente a los procesos en marcha de manera eficaz y coordinada. En esta fecha aparecen las estructuras básicas de un Departamento (Direcciones Generales) que ponen de manifiesto la pretensión fundamental de construir un Ministerio homologado con el resto de los Departamentos de la Administración. Es pre- 
cisamente ese esfuerzo de homologación, en combinación con las especificidades de la administración militar, lo que concentra uno de los mayores esfuerzos del proceso de reforma, porque el problema no fue sólo recabar competencias que hasta entonces desarrollaban los ejércitos, sino también ejercerlas hacia adentro con rigor y en términos de coordinación, pero hacia el exterior poniendo de manifiesto que los elementos comunes de una administración moderna deben cohonestarse con los específicos de la administración militar, algunas de cuyas pautas son propias. Esto es lo que significa el esfuerzo de homologación más especialidad. Concebida desde el principio como una administración dinámica, la estructura del Departamento fue recibiendo un impulso que se concretó en sucesivas reformas que siguen los parámetros indicados al menos hasta 1996.

La disposición de una estructura potente permitió llevar adelante procesos sustanciales de reforma y modernización de las Fuerzas Armadas que en otro caso hubiera sido muy complejo arbitrar. Uno de ellos es el proceso de planeamiento, el control presupuestario y el régimen de las adquisiciones; otro la política de personal, muy relevante, pero especialmente difícil de poner en práctica sobre todo en términos de dimensionamiento de los ejércitos, de regulación de la carrera militar y de adaptación de los sistemas de enseñanza a un modelo global, abierto e integrado en el sistema general del Estado.

Hasta aquí la referencia a la dirección política de la defensa. Los órganos militares, por su parte, son de carácter técnico, es decir, ejercen una función ejecutiva. Están fundamentalmente constituidos por los Jefes de Estado Mayor, a quienes corresponde, bajo la autoridad del Ministro, el mando de sus respectivos ejércitos. Cada Jefe de Estado Mayor se relaciona directamente con el Ministro sin la intermediación de la Junta y, en la primera etapa, también sin la interposición del Jefe del Estado Mayor de la Defensa.

Esta figura merece alguna referencia especial. Inexistente hasta 1984, aparece en la Ley reformada como el principal colaborador del Ministro en el planeamiento y ejecución de los aspectos operativos de la política militar. Su integración no es sencilla, pues constituye un escalón intermedio en la jerarquía del mando que resulta de difícil encaje y que viene a ser percibido como un órgano que sustrae competencias al Jefe del Estado Mayor del correspondiente ejército. El cuidadoso diseño de la figura, muy incipiente, en la Ley de Criterios básicos, ha dejado paso a la definición de un órgano con perfiles más delineados y llamado a realizar una función cada vez de mayor entidad. Va asumiendo competencias sucesivamente en la acción conjun- 
ta y en la acción operativa, en la que todavía a comienzos de 1996 se le atribuyen capacidades específicas ante la necesidad de responder con un mando operativo eficaz en los cada vez más complejos despliegues de tropas en escenarios externos. La nueva Revisión Estratégica aún pretende dar un paso adelante en la definición de esta figura especialmente dinámica en nuestro modelo de defensa.

El segundo principio informador de la reforma, la personalización de las decisiones, equivale, en mi criterio, a la atribución de capacidades de dirección en cualquiera de los niveles, político y técnico, a órganos unipersonales. Dicho de otro modo, se refuerzan, por una parte, los órganos unipersonales de dirección política $y$, de otra, los órganos unipersonales de ejecución. Todo ello en detrimento de los órganos colegiados que, como se ha dicho, tienen fuertes competencias hasta 1984 y que ahora pasan a desempeñar una función puramente asesora. Así venía sucediendo ya con la Junta de Defensa Nacional, que es órgano superior consultivo del Gobierno en materia de defensa nacional y que asesora al Presidente y al Jefe del Estado (y que, nuevamente según la Revisión Estratégica, se convertirá en un Consejo de Seguridad y de Defensa nacional), pero a partir de la fecha indicada también con la Junta de Jefes de Estado Mayor en los términos antedichos. Permanecen competencias residuales, aunque importantes, en los Consejos Superiores de cada ejército. Determinada crisis relacionada con la renovación de la Junta de Jefes de Estado Mayor motivó su conversión en órganos puramente asesores en el año 1985 (Real Decreto-Ley 3/1985, de 10 de julio).

Finalmente, aunque la articulación entre defensa y poder judicial tiene pautas diversas, es preciso hacer referencia en este apartado, para completar el cuadro y sin perjuicio de lo que luego se dirá, al tercero de los poderes del Estado. La Constitución se limita a establecer un mandato de unidad jurisdiccional (art. 117) que ya la Ley de Criterios básicos de 1980 pretende concretar a través de la reforma de las leyes penales militares.

No obstante, no es sino hasta 1985 cuando comienza un proceso de revisión que se desarrolla a través de un paquete de varias normas con rango de ley y que se prolonga durante cuatro años. Se opta por un sistema de reforma jurisdiccional que mantiene la especificidad de la jurisdicción castrense, pero que da cumplimiento pleno al mandato de unidad jurisdiccional. Dos son, en mi criterio, los principios que pueden destacarse como fundamentos del proceso: la separación entre mando y jurisdicción y la integración en el poder judicial único del Estado del sistema jurisdiccional militar. 
En base a ellos, la función de juzgar, distinta de la función de mando, que es ejecutiva, queda exclusivamente atribuida a los órganos judiciales, mientras que a la función de mando corresponde la potestad disciplinaria. Se mantiene una estructura jurisdiccional propia, pero profesionalizada, cuyo primer escalón son los Juzgados Togados y que distribuye la competencia entre un Tribunal central y unos Tribunales territoriales de carácter permanente y compuestos por juristas militares y por oficiales de los ejércitos elegidos a modo de escabinos. La unidad jurisdiccional se sitúa en el vértice de la jurisdicción con la institución en el Tribunal Supremo de una Sala de lo Militar que cuenta con una composición específica: la mitad de sus componentes procede del Cuerpo Jurídico militar, aunque pierden su condición de tal al pasar al Tribunal Supremo.

\section{OBJETIVOS DE DEFENSAY MISIONES DE LAS FUERZAS ARMADAS}

El análisis que se llevado a cabo en el apartado anterior pone de manifiesto la imbricación de las fuerzas armadas en el entramado de los poderes del Estado. Queda, pues, fijado el modelo de integración de los ejércitos en el ámbito de la autoridad pública, que es un elemento fundamental de lo que he denominado proceso de institucionalización de la defensa, que responde a una preocupación prioritaria del momento constitucional y que, como se ha visto, se desarrolla de una manera compleja y a lo largo de un tiempo prolongado.

Queda aún una segunda vertiente por explicar, porque dicho proceso se fundamenta también en la definición dinámica del quehacer de los ejércitos. Así pues, la cuestión no es sólo quién ejerce la autoridad, sino también qué deben hacer las Fuerzas Armadas o, lo que es lo mismo, cómo se concretan las misiones constitucionales.

El gran debate en este campo es la determinación de las misiones que compete realizar a los ejércitos, sin suplantar las funciones de otras instituciones del Estado. No es cuestión exenta de problemas en la actualidad; mucho menos lo fue en la perspectiva de la vertebración de la sociedad española que se emprende desde la transición. Desde un criterio sistemático, creo que la reflexión sobre esta segunda vertiente del mencionado proceso puede realizarse con atención a una doble óptica, estática y dinámica. La primera es una perspectiva de contención y hace referencia al apartamiento de las estructuras militares del ejercicio de funciones que no les son propias; la segunda es 
una visión en positivo, es decir, la definición de funciones según los objetivos a alcanzar. La segunda perspectiva no es posible sin la primera y es preciso indicar que aquélla, más evolucionada, suele responder a un momento de progresiva madurez en el proceso de institucionalización de la defensa.

En el modelo español son distinguibles, estimo, las dos perspectivas que, sin embargo, corren en paralelo alguna parte de su camino. Desde luego, la definición en positivo de la política de defensa española no se emprende hasta mediados de los ochenta. Hay en 1982. un dato de interés. Un año antes se había promulgado una Directiva de Defensa Nacional que se limitaba a incluir las clásicas referencias estratégicas de las que se ha dicho que permanecían ancladas en las concepciones tradicionales. Pero en 1982, en medio de una fuerte polémica, se produce el ingreso de España en la OTAN. No hay, sin embargo, una doctrina estratégica propiamente dicha bajo esta decisión, más allá la política de bloques. Un analista francés ha dicho atinadamente que para la perspectiva española, mucho más que este criterio pesaba la incorporación a los valores occidentales compartidos de todos los sectores del pais, incluyendo los ejércitos.

En 1986 se celebra el comprometido referéndum para la permanencia en la Alianza Atlántica. Habia avanzado algo para esa fecha la definición de la política de defensa, como se dirá a continuación. Pero, sobre todo, ya se había emprendido una línea de la reforma militar en la mencionada perspectiva de la contención. Fruto de este planteamiento es la reducción sustancial, siguiendo de nuevo el mandato de la Constitución, de la jurisdicción castrense. En su virtud, el ámbito del Derecho penal militar (del que, por cierto, ya había desaparecido el delito de rebelión militar como resultado del intento de golpe de Estado de 23 de febrero de 1981 para pasar al Código penal común) queda reducido a los delitos militares. Desaparece la impropia extensión de la jurisdicción militar por la vía del triple fuero para fijar su competencia sólo en las conductas delictivas tipificadas en una ley penal de 1985 que se elabora con criterio restrictivo. A partir de ese momento, las fuerzas armadas dejan de participar por la vía jurisdiccional en la represión de conductas que nada tienen que ver con los aspectos militares.

Desde un punto de vista sustantivo, se promulga en 1986 la Ley de Fuerzas y Cuerpos de seguridad del Estado que traza una línea clara de separación entre las funciones militares y las de mantenimiento del orden público. No sin debate, a causa de la naturaleza militar que la Ley atribuye al Cuerpo de la Guardia civil y también por la estra- 
tegia de tensión que el terrorismo viene poniendo en práctica, se establece un sistema complejo de distribución de las competencias que, sin embargo, asegura la definitiva separación de los ejércitos del desarrollo de labores que corresponden a las fuerzas de seguridad del Estado.

La definición de los objetivos de la defensa nacional, por su parte, había dado un paso adelante en 1984 con la primera Directiva de Defensa Nacional del nuevo Gobierno. Estos objetivos no están aún del todo depurados, pero ya introducen un cambio de perspectiva transcendental: el abandono del concepto de defensa interior como misión de los ejércitos, que quedan orientados, aunque ciertamente de un modo que hará necesaria la insistencia de posteriores Directivas, hacia la defensa de la agresión exterior y la colaboración con los ejércitos aliados. Este planteamiento se completa, el mismo año, con la apertura del primer debate parlamentario sobre política de defensa $y$ seguridad. El Presidente del Gobierno presentó un conjunto de diez medidas (Decálogo, se llamó) que son una expresión más depurada de las posiciones españolas sobre la OTAN, relaciones con Estados Unidos, cooperación bilateral, foros de desarme o presencia de armas nucleares en el territorio. De estas medidas, las cuatro primeras constituyeron el cuerpo del referéndum sobre la Alianza Atlántica, que se celebra en febrero de 1986.

A partir de este momento, la progresiva consolidación del sistema permite pensar ya en una definición más rigurosa de la política de defensa que se elabora mediante la profundización en las líneas avanzadas en 1984. El proceso continúa y en 1988 España ingresa, junto con Portugal, en la Unión Europea Occidental; en el mismo año, con cierta timidez, se inician las primeras operaciones por cuenta de Naciones Unidas en el continente africano. Algunos autores señalan que a partir de los años noventa, la política de defensa española deja de estar condicionada por los elementos internos. La fecha es anterior. Pero sí es cierto que entramos en un proceso que se ha denominado de desterritorialización como un paso más desde el inicio de la apertura al exterior de la política de defensa española.

\section{EL CAMBIO DEL ESCENARIO ESTRATÉGICO}

Al final de la década de los ochenta el mundo ya había comenzado a sufrir cambios profundos. Alguien se atrevió a decir, ciertamente con pocas dosis de acierto, que el siglo, en este campo, termi- 
nó diez años antes. Aún quedaba otro cambio radical en la seguridad del mundo globalizado con el final verdadero del siglo. Pero desde el comienzo de la última década del siglo $X X$ ya no es posible entender la defensa como un complejo dispositivo militar que se actúa para repeler una agresión. Las circunstancias han sufrido cambios tan sustanciales que obligan a conformar sobre nuevas bases las doctrinas de seguridad y defensa. La seguridad sufre amenazas nuevas, la defensa se globaliza. En este contexto, las Fuerzas Armadas acercan cada vez más su naturaleza al carácter de instrumentos de la política internacional del Estado. La referencia internacional obliga a desarrollar nuevas capacidades que exigen un gran esfuerzo a la organización. Desde mi perspectiva, el gran cambio en el escenario mundial ha podido afrontarse precisamente porque el proceso del que se ha querido dar cuenta en las páginas anteriores situó a los ejércitos españoles y a la propia administración militar en condiciones de poder llevarlo a cabo. Pero puede entenderse que a partir de este momento se produce también un cambio subsiguiente en el proceso de que se está dando cuenta.

Pues bien, en el nuevo escenario estratégico se trata de salir al paso de riesgos diversos y multiformes que no siempre suponen en si mismos una grave amenaza, pero cuyas repercusiones ponen en peligro los intereses de la nación. Las nuevas circunstancias de los años noventa, entre las que las más determinantes son la caída del Muro, la desmembración de la Unión Soviética, la disolución del Pacto de Varsovia y la guerra del Golfo, provocan el aludido cambio radical. Esta es la idea de la que parte una nueva Directiva de Defensa Nacional de marzo de 1992.

Con anterioridad a ella, pero en proceso paralelo, se había producido en España la reflexión parlamentaria, ya mencionada, sobre las Fuerzas Armadas para el final del siglo que ya integra el nuevo escenario estratégico y que determinó un modelo de ejércitos más reducido, mejor tecnificado y sensiblemente profesionalizado: un ejército mixto.

La nueva Directiva, como se ha dicho, llegó en paralelo a este proceso. Su contenido es ya mucho más exacto y moderno. Los Objetivos de la defensa nacional, con los obligados de garantía de la soberanía y de los intereses vitales de la nación, se sitúan decididamente en el campo de la seguridad colectiva, en el terreno de los compromisos internacionales y en el ámbito de la colaboración en el fortalecimiento de las relaciones pacíficas entre las naciones. Estos contenidos se han sistematizado gráficamente como objetivos de carácter 
nacional, regional y global. Pero lo verdaderamente significativo es la decidida vinculación de la seguridad española con los esquemas de la seguridad colectiva. Es precisamente esa idea de seguridad compartida con las naciones que forman parte de nuestro entorno cultural y que defienden un sistema basado en los valores de la democracia y del Estado de Derecho, lo que refleja más fielmente la línea aperturista de la política de defensa española.

La concreción más evidente es el alcance que obtienen las intervenciones de los ejércitos españoles en misiones de paz y ayuda humanitaria a partir de este tiempo. Se llevan a cabo operaciones en todos los continentes y de manera prácticamente continua. De entre ellas destaco por vinculación especial las desarrolladas en Centroamérica y por entidad las de la antigua Yugoslavia.

Sobre los avances de la Directiva de 1992, se construye tres años más tarde una formulación dinámica y permanentemente abierta de la política de defensa. En el documento que se envía por el ejecutivo en el mes de junio al Congreso de los Diputados se insiste tanto en fortalecer la conciencia de defensa nacional cuanto en el impulso a la integración europea, a la participación en la adaptación de la OTAN a las nuevas estructuras y en las operaciones de paz. Es la última acción en la materia de los gobiernos del Partido Socialista.

El nuevo gobierno del Partido Popular emite en 1996 una nueva Directiva de Defensa Nacional que incorpora a los planteamientos existentes algunos nuevos datos procedentes de la culminación de los procesos en marcha. Estos nuevos datos son, por una parte, la plena incorporación a la Alianza Atlántica, aprovechando la definición de las nuevas estructuras de la organización para incorporar los nuevos requerimientos estratégicos y, sobre todo, la profesionalización de los ejércitos sobre la base política de la crisis del servicio militar que se había detectado mucho antes. Este último supuesto es ciertamente, más que un precipitado del nuevo escenario internacional, un asunto de politica interna.

El dato más relevante es justamente este último. Desde una perspectiva dinámica, una de sus referencias es el proceso de redimensionamiento continuo de las Fuerzas Armadas emprendido desde 1982. Más específicamente, es el paso definitivo con respecto de la definición de un modelo mixto que se había llevado a cabo en 1991. La plena profesionalización, como se ha dicho anteriormente, se decide por el Gobierno en 1996 y se estudia mediante una comisión parlamentaria que emite su informe dos años más tarde. En este momento las Fuerzas Armadas españolas son profesionales. Será necesario solu- 
cionar algunos problemas de encaje $y$, especialmente, resolver los problemas más graves que están ligados al reclutamiento.

Este es el marco de la nueva ordenación de la defensa. Desde un planteamiento estratégico compartido con nuestros aliados y que busca la compenetración con los países vecinos, pero también con aquellos que están situados en las áreas de interés estratégico, se formulan Objetivos que tienden a consolidar la presencia de España en las organizaciones internacionales, a capacitar a las Fuerzas Armadas para la defensa colectiva y para las misiones internacionales de mantenimiento de la paz y para desarrollar la conciencia de defensa nacional entre los ciudadanos como un elemento necesario para alcanzar los objetivos anteriores.

Este largo proceso de definición de los objetivos de la defensa española no termina aquí. Hoy está vigente un nuevo marco establecido por la nueva Directiva de finales de 2000. En esa fecha se fijan como objetivos prioritarios tres elementos principales que marcan, al menos los dos primeros, el sentido de las misiones de las fuerzas armadas. El primero es garantizar la seguridad y defensa de España y de los españoles en el marco de la seguridad compartida y de la defensa colectiva. El segundo, contribuir a las misiones de ayuda humanitaria y de operaciones de paz y de gestión de crisis que realicen las organizaciones internacionales y europeas a las que España pertenece. $Y$ el tercero se refiere a fomentar la conciencia de defensa nacional en la sociedad española a través de la cultura de defensa.

Estos objetivos requieren una concreción que se aborda a través de un conjunto de líneas básicas especificadas en la propia Directiva para abordar de forma integrada las cuestiones «orgánicas, funcionales y operativas" que se implican en la defensa. Entre ellas, se hace referencia a las exigencias de la consolidación del nuevo modelo de ejércitos profesionales que se ha implantado en España; también a la modernización y organización de las estructuras de la defensa para garantizar la interoperabilidad y la acción conjunta. Pero me permito destacar, como más novedosa, la intención de iniciar una Revisión estratégica que permita, siguiendo el modelo de algunos países, reformular la concepción estratégica española para los próximos diez o quince años.

Se trata, ciertamente, de un planteamiento ambicioso. Requerirá, en primer lugar, determinar los posibles escenarios, intereses y riesgos de la política estratégica española. También la definición de las misiones de las Fuerzas Armadas españolas en el nuevo orden internacional y, en función de ellas, definir sus necesidades futuras, caren- 
cias y requerimientos para su organización funcional. Es un problema real enfrentar el análisis de una situación estratégica tan fluida como la actual con horizontes de tiempo tan amplios, pero parece conveniente la fijación de unos criterios tendenciales que sirvan de referencia para la acción política. Esta formulación debe tener un componente dinámico que permita ir integrando en sucesivas revisiones las nuevas circunstancias requeridas por los cambios sociales.

La citada Revisión Estratégica se integra en la reflexión acerca de una nueva seguridad compartida y establece unas misiones para las fuerzas armadas que son, hoy por hoy, la meta de un largo proceso. Distingue, por un lado, unas misiones defensivas: impedir la agresión y, si es el caso, responder a ella incluso en el exterior en el marco de las organizaciones internacionales. De otra parte, se asigna a los ejércitos la misión de contribuir a la paz y seguridad internacionales, que se concretan en misiones de cooperación internacional. Finalmente, se establece la contribución militar a la seguridad y bienestar de los ciudadanos mediante el apoyo a las políticas que desarrollen otras administraciones, en situaciones de emergencia $y$ otras. Sin duda queda un camino por delante para concretar el desarrollo de algunas de estas funciones. También para definir la participación de los ejércitos en cuestiones relacionadas con la lucha contra el terrorismo global, que la Revisión resuelve mediante la referencia al terrorismo exterior. Pero es notable el esfuerzo de convergencia de los grupos políticos en la definición de estos criterios estratégicos.

\section{LA CONSTITUCIÓN, MOTOR DE LA REFORMA. UNA CONSTITUCIÓN ABIERTA}

Con la perspectiva, en fin, de esta panorámica, pareciera que la Constitución de 1978 queda muy lejana en el tiempo. El cuarto de siglo de su vigencia cobijó esfuerzos muy diversos para la adaptación de las Fuerzas Armadas al sistema de convivencia en ella definido y hasta un cambio radical en la naturaleza de los problemas. En esta reflexión esencial se ha querido poner el acento en una doble perspectiva: en primer lugar, la construcción de una base sólida para el sistema; en segundo lugar, la disposición o cambio de óptica con que deben enfrentarse los nuevos problemas.

Son distinguibles, desde este punto de vista, dos fases en el proceso. La primera se abre con la propia Constitución, que pacifica un 
problema histórico a fuerza de consenso y apunta las soluciones que deben arbitrarse para el enfrentamiento con las cuestiones básicas que plantea. La preocupación fundamental es lo que antes se ha llamado constitucionalización de las Fuerzas Armadas. Para responder a ella, el texto constitucional requiere las reformas (así pues, es motor del proceso) y las encauza en un camino de márgenes flexibles. La reforma militar, siguiendo las preocupaciones constitucionales, se aplica al proceso de institucionalizar la defensa. La definición del papel de los poderes públicos en la dirección y control de la defensa y el establecimiento de los criterios de funcionalidad de los ejércitos son los elementos clave de dicho proceso.

Esta labor se ha realizado a través de un camino largo y complejo, que se acusa especialmente desde 1984, que trabaja en campos diversos, con el concurso de los poderes públicos en su conjunto y a través de disposiciones situadas en diversos niveles de la jerarquía normativa. La dinamicidad del propio proceso obliga a trabajar con programas de actuación esenciales y atentos a la incorporación de las respuestas a las necesidades que se van planteando.

Entrada la década de los noventa, eclosiona el cambio de circunstancias que se anunciaba desde el final de la década anterior. Con la mirada puesta en el nuevo escenario estratégico, se abre una fase de despegue para la política de defensa española. Atrás queda un sólido proceso de institucionalización de.los ejércitos, de fijación de los objetivos básicos y de las misiones de las Fuerzas Armadas, así como de la implantación de los grandes hitos de la reforma: la regulación de la carrera militar, la reforma de la enseñanza, la programación, el modelo mixto.

A partir de entonces, el gran reto es disponer de la flexibilidad necesaria para adaptarse a las exigencias de tiempos que evolucionan vertiginosamente en doctrina de seguridad y que plantean respuestas cada vez más exigentes en busca del bienestar de los ciudadanos. El sistema institucional de la defensa que se había construido permitió enfrentarse al nuevo momento histórico con capacidad de adaptación suficiente.

Así pues, la Constitución parece alejada de las actuales preocupaciones en este campo, pero ha sido motor, palanca del proceso de reforma $y$ un instrumento especialmente apreciable como referencia y cauce de los momentos fundamentales del proceso. Ha tenido el mérito de diseñar un modelo esencial de incorporación de las Fuerzas Armadas al sistema de convivencia y hacerlo desde una visión com- 
partida por todos. Por esta razón ha sido capaz de cobijar los requerimientos complejos del proceso que se ha presentado. Por esta razón, también, no limita posibilidades de futuro, antes bien, sienta un sistema abierto con la referencia de unos principios fundamentales que lo ordenan. De ahí que para integrar en el ordenamiento las preocupaciones emergentes para la política de seguridad y defensa sea suficiente la adaptación de otras disposiciones, como la Ley de Criterios básicos de la Defensa nacional, que informen la actuación concreta de las Fuerzas Armadas. 